\title{
Resuscitation interventions in a tertiary level pediatric emergency department: implications for maintenance of skills
}

\author{
F. Jonathan Guilfoyle, $\mathrm{MD}^{*}$; Ruth Milner, $\mathrm{PhD}^{\dagger}$; Niranjan Kissoon, $\mathrm{MD}^{\ddagger}$
}

\section{ABSTRACT}

Objective: To describe the frequency and proportion of successful resuscitation interventions in a pediatric emergency department (PED).

Methods and Material: This was a retrospective chart review of children at the BC Children's Hospital (BCCH) PED who were admitted to the $\mathrm{BCCH}$ pediatric intensive care unit (PICU) in 2004 and 2005. Demographic data, diagnosis, and resuscitation interventions in the PED and within the first 24 hours of PICU admission were recorded. The training of the operator and the number of attempts needed were also recorded.

Results: There were 75,133 PED visits; 304 of 329 (92.4\%) who met inclusion criteria were reviewed. Diagnoses included respiratory distress $(n=115,35 \%)$, trauma $(n=50,15 \%)$, sepsis ( $n=36,11 \%)$, seizures ( $n=37,11 \%)$, and cardiac disease ( $n=22,7 \%$ ). Ninety-nine patients required intubation. Intubations in the PED were performed by residents $(20 \%)$, pediatric emergency medicine (PEM) fellows $(15 \%)$, PEM attending staff (29\%), and PICU fellows (12\%); $81 \%$ of these were successful on the first attempt. In the PED, seven central lines were placed, seven intraosseous needles were inserted, 15 patients required inotropes, and 9 patients required chest compressions.

Conclusion: Critical illness in our emergency department is a rare event; hence, opportunities to resuscitate, secure airways, and place central venous catheters are limited. Additional training, close working relationships between the PED and the PICU teams, and resuscitation protocols for early PICU involvement may be needed.

\section{RÉSUMÉ}

Objectif: Décrire la fréquence des manœuvres de réanimation et le taux de succès dans un service des urgences pédiatriques (SUP).
Méthode et matériel: Nous avons réalisé une étude rétrospective des dossiers d'enfants s'étant présentés au SUP de I'Hôpital pour enfants de la Colombie-Britannique $(\mathrm{BCCH})$ et ayant été admis à I'Unité des soins intensifs pédiatriques (USIP) de cet hôpital en 2004 et en 2005. Les données démographiques, le diagnostic et les manœuvres de réanimation réalisées au SUP et dans les 24 premières heures suivant I'admission à I'USIP ont été consignés en dossier. La formation de l'opérateur et le nombre de tentatives nécessaires ont également été consignés en dossier.

Résultats: II y a eu 75133 visites à l'urgence des soins pédiatriques; 304 des 329 visites (92,4\%) répondant aux critères d'inclusion ont été analysées. Les diagnostics comprenaient : détresse respiratoire $(n=115,35 \%)$, traumatismes ( $n=50,15 \%)$, sepsis $(n=36,11 \%)$, convulsions ( $n=37,11 \%$ ), et maladie cardiaque ( $n=22,7$ $\%)$. Quatre-vingt-dix-neuf patients ont nécessité une intubation. Les intubations au SUP ont été réalisées par les résidents $(20 \%)$, les moniteurs cliniques (fellows) en médecine d'urgence pédiatrique (15\%), les médecins traitants du SUP (29\%), les moniteurs cliniques à I'USIP $(12 \%)$. Le taux de succès de ces intubations à la première tentative était de $81 \%$. Au SUP, 7 cathéters centraux et 7 abords veineux intra-osseux ont été mis en place, des inotropes ont été administrés à 15 patients et des compressions thoraciques pratiquées chez 9 patients.

Conclusion: Des cas de maladies graves sont rares à notre service d'urgence. Les occasions de pratiquer des manœuvres de réanimation, d'ouvrir les voies aériennes et de poser un cathéter central sont donc limitées. II faut envisager une formation supplémentaire, des relations de travail étroites entre les équipes du SUP et de I'USIP et la mise en place de protocoles de réanimation pour une intervention précoce de I'USIP.

From the *Department of Paediatrics, Division of Emergency Medicine, Alberta Children's Hospital, Calgary, AB; †Dept of Paediatrics and Division of Emergency Medicine, University of British Columbia, Vancouver, BC; and $¥ B C$ Children's Hospital and Child and Family Research Institute, Vancouver, BC.

Correspondence to: Dr. Niranjan Kissoon, BC Children's Hospital, 4480 Oak Street, Room B245, Vancouver, BC V6H 3V4; nkissoon@cw.bc.ca.

Submitted October 23, 2009; Revised February 6, 2010/ February 14, 2010; Accepted February 21, 2010

This article has been peer reviewed.

(c) Canadian Association of Emergency Physicians

CJEM 2011;13(2):90-95

DOI 10.2310/8000.2011.110230 
Keywords: children, critical illness, emergency, endotracheal intubation, epidemiology, intensive care, intraosseous cannulation, resuscitation, simulation, vascular access

Critical illness in the pediatric emergency department (PED) is rare. A review of the National Registry of Cardiopulmonary Resuscitation found that of the 23,535 in-hospital arrests recorded over a 2-year period, only $778(3.3 \%)$ occurred in children. ${ }^{1}$ Of those in children, only $8.9 \%$ occurred in the PED. ${ }^{1}$ More recently, a study in a large tertiary level PED reported that residents' exposure to critical illness and their opportunities to perform lifesaving interventions were very limited. ${ }^{2}$ We found no studies that examined all of the resuscitation interventions performed in a PED.

Based on the limited data available and our clinical experience, we hypothesized that critical illness is rare in our PED and opportunities to perform resuscitation interventions (eg, intubations, central line placement, tube thoracostomy, cardiopulmonary resuscitation [CPR], and use of inotropes) are limited for both trainees and attending physicians. Ultimately, we hoped that by quantifying the number of opportunities for trainees and attending physicians to perform resuscitations, we could estimate whether their training needs for such skills were being met by their clinical time spent in the PED. Thus, the main goal of this study was to quantify the type and number of resuscitation interventions in a tertiary level PED, and the level of training and proportion of success of those performing these interventions. We also discuss the relevance of these findings for maintenance of skills for resuscitation.

\section{METHODS}

\section{Setting}

This was a retrospective chart review of critically ill children at a PED in an urban children's hospital that has 37,500 visits annually. The PED is served by 17 pediatric emergency medicine (PEM) attending staffs, 7 PEM fellows, and 7 to 12 rotating residents per month. The hospital has a 22-bed pediatric intensive care unit (PICU) with in-house coverage by PICU fellows, senior residents, and clinical assistants.

\section{Case Identification}

Inclusion criteria consisted of all children who presented to the PED and were subsequently admitted to the PICU or those who died in the PED over 2 years (January 1, 2004, to December 31, 2005). Patients who were transferred directly to the PICU from other facilities and patients admitted to other units within or outside the hospital were excluded.

\section{Chart Abstraction}

Cases were identified using a preexisting, prospective PICU database designed to capture data on all aspects of care, including demographics, Canadian Triage and Acuity Scale (CTAS) scores, diagnosis, procedures, length of stay (LOS), and outcomes. The data, which are used for quality assurance purposes, were collected on admission and the charts were reviewed again, on discharge, by an experienced PICU nurse employed and trained for this purpose. The database was used to generate a list of the 323 patients admitted to the PICU from the PED. The intent was to review all 323 charts; however, owing to challenges in retrieving charts stored off-site and in subspecialty clinics, 298 charts $(92 \%)$ were fully reviewed and included in this study. The PICU database allowed for the identification of patients who were intubated, and all of these patients were included in the 298 reviewed charts. A PED database provided a list of six patients who died in the PED and were thus never admitted to the PICU. All six of these charts were reviewed.

\section{Review Methods}

The charts were reviewed by three abstractors, consisting of two senior emergency department (ED) trained research nurses and one senior medical student, who were trained in chart abstraction and oriented to the abstraction form by the lead investigators. The abstractors were not blinded to the study objectives. Scheduled and informal meetings were held regularly to resolve any issues with chart abstraction and coding as they developed. The abstractor's performance was closely monitored by the lead investigators to ensure that the data were collected accurately. Although there was no formal assessment of interrater reliability, the majority of charts were reviewed by two senior research nurses who worked closely together and regularly reported any discrepancies or challenges to the investigators. The data from the abstraction forms were then entered into a spreadsheet for data analysis, and 
during this process, charts were reviewed again if discrepancies or missing data were noted.

\section{Data Management}

Relevant data were collected using a standard abstraction form, designed by the authors, which coded for the interventions common in a resuscitation (intubations, central line placement, intraosseous [IO] needle insertion, chest tube insertion, and chest compressions) along with the number of attempts and level of training of the operator. For the purpose of this study, the definition of a failed procedure was limited to documentation in the medical record of a failed or a second attempt. The form allowed for uncoded data to be collected under "other" and included codes for missing or unknown data.

We also recorded the type and number of resuscitation interventions during the first 24 hours of PICU admission and eventual disposition. We did not, however, include the level of training and success rate of those performing intubations. Statistical analysis was limited to descriptive data, including mean and standard deviation or median and interquartile range, as appropiate.

\section{Ethics}

Ethics approval was obtained from the University of British Columbia and the Institutional Ethics and Patient Safety Review Board.

\section{RESULTS}

During the 2-year study period, 75,133 patients visited the PED, of whom $323(0.3 \%)$ were admitted to the PICU. The charts of 298 (92\%) of these admissions were reviewed. During the study period, six patients were pronounced dead in the PED, and their charts were also reviewed. A total of 304 charts were reviewed.

Patients presented to the PED with a variety of diagnoses, the most common of which was respiratory illness, followed by trauma and sepsis. The average age was 5.3 years, and 55 (\%) were boys; the median CTAS score was 2 (Table 1 ).

In the PED, 58 children required endotracheal intubation. Seven patients had an IO needle inserted,

\begin{tabular}{lc|}
\hline Table 1. Patient demographics & \\
\hline Patient demographics & $n(\%)$ \\
\hline Average age (yr) & 5.3 \\
Sex (\% boys) & 55 \\
ED presentations & 2 \\
$\quad$ Median CTAS score & \\
Disposition from PED & 323 \\
Admission to PICU & 304 \\
Charts reviewed & 6 \\
Pronounced dead in ED & \\
Disposition from PICU & $267(90)$ \\
Discharged home & $17(6)$ \\
Transfer to another facility & $11(4)$ \\
Died & $3(1)$ \\
Unknown & $115(35)$ \\
Diagnostic categories & $50(15)$ \\
Respiratory illness & $37(11)$ \\
Trauma & $36(11)$ \\
Seizures & $22(7)$ \\
Sepsis & $8(2)$ \\
Cardiac disease & $53(16)$ \\
Toxins & \\
Other & \\
\hline CTAS = Canadian Triage and Assessment Scale; ED = emergency department; PED $=$ \\
pediatric emergency department; PICU = pediatric intensive care unit.
\end{tabular}

four of whom were moribund and pronounced dead in the PED. Other interventions performed in the PED, such as central line placement, chest tube insertion, and chest compressions, were uncommon. Most children only required fluid bolus for cardiovascular support, and inotropes were rarely necessary (Table 2).

Of the 58 intubations performed in the PED, $81 \%$ $(n=47)$ were successful on the first attempt, $10 \%$ $(n=6)$ on the second attempt, and 3\% $(n=2)$ on the third attempt. Intubations in the PED were performed by PEM attending staff (29\%), PEM fellows (15\%), residents $(20 \%)$, and PICU fellows (12\%); the person performing the intubation was not documented in $19 \%$ of cases. The first attempt success rate of PED and PICU fellows was $100 \%$, of PED attending staff was $76 \%$, and of residents was $67 \%$. On average, intubation was performed 82 minutes after arrival at the PED; a third $(n=19)$ of patients required intubation within 30 minutes, and $14 \%(n=8)$ required intubation within 10 minutes.

In the PICU, 41 patients were intubated within 24 hours of admission (14\%). The time of intubation was recorded in $63 \%(n=26)$ of cases and was, on average, 7.5 hours after PICU admission; however, $15 \%(n=4)$ occurred within 30 minutes and $38 \%(n=10)$ within 


\begin{tabular}{|lc|}
\hline \multicolumn{2}{|l|}{ Table 2. Resuscitation interventions in the PED and PICU } \\
\hline Intervention & $n(\%)$ \\
\hline PED & $119(39)$ \\
Fluid bolus infusion & $58(19)$ \\
Intubations & $15(5)$ \\
Inotropic support & $9(3)$ \\
Chest compressions & $7(2)$ \\
Intraosseous cannula insertion & $7(2)$ \\
Central line insertion & $6(2)$ \\
Albumin bolus infusion & $6(2)$ \\
Blood transfusion & $2(0.6)$ \\
Chest tube insertion & \\
PICU & $41(14)$ \\
Intubations & $37(12)$ \\
Central line insertion & $27(9)$ \\
Inotropic support & $1(0.3)$ \\
Chest tube insertion & $0(0)$ \\
Chest compressions & \\
\hline PED = pediatric emergency department; PICU = pediatric intensive care unit.
\end{tabular}

2 hours of admission. Inotropic support was required in 27 patients. Central line placement was performed in $10 \%$ of the patients admitted to the PICU. Chest tube insertion was performed once, and there was no documentation of chest compressions being performed (see Table 2).

The average LOS in the PICU was 4.8 days; the total hospital LOS was 11.6 days. Most children $(n=$ $269,90 \%)$ were eventually discharged home, 17 (6\%) were transferred to another hospital or a long-term care facility, and 11 (4\%) died; only 3 (1\%) charts had missing outcome information.

\section{DISCUSSION}

We conducted this study because of the lack of data documenting the number of resuscitation interventions performed in the PED and the concern that there may be insufficient exposure to acquire and maintain these skills. ${ }^{3}$ Our results confirm that critical illness and related interventions occur infrequently in our PED, and opportunities for trainees and attending physicians to perform such procedures are limited. Our results are similar to those of a PED of a large children's hospital, which found that only 2.5 patients per 1,000 visits required treatment in a resuscitation room. ${ }^{4}$ Our data are also consistent with studies that found a low volume of resident exposure to critical illness and resuscitation interventions in the $\mathrm{PED}, 2,5$ as well as the National Registry of Cardiopulmonary Resuscitation, which found that CPR in the PED was exceedingly rare. ${ }^{1}$ Therefore, our experience in a midsized tertiary care ED is not unique; however, this is the first such study from a Canadian PED site.

The importance of airway management in the PED is highlighted by the fact that most pediatric arrests are respiratory in origin, with cardiac arrest as a consequence of inadequate respiratory support. ${ }^{4,6}$ In our PED, intubation was a rare event, with a rate of 0.9 per 1,000 patient visits, which is similar to the rates (1.1$3.3 / 1,000)$ in similar centres. ${ }^{7}$ With an average of 29 intubations a year and a PED staffed by 17 PEM attending physicians, 7 PEM fellows, and 7 to 12 rotating residents per month, there are very limited opportunities to practice this essential skill. PEM fellows are often given priority to perform intubation over other trainees. However, for many emergency medicine, pediatric, and family medicine residents, who will eventually staff community EDs, where most ill and injured children are initially assessed and stabilized, their rotation through the PED may be one of their few exposures to critically ill children and may be their only opportunity to practice intubation with supervision before facing the challenge of securing a pediatric airway in a peripheral centre with limited backup.

Despite the relatively infrequent occurrence of intubations, the overall success rate of intubation on the first attempt $(81 \%)$ is comparable to that of other studies in which first attempt success rates range between 80 and $83 \% .^{8-10}$ The lowest success rate $(67 \%)$ was, as expected, in the resident group; however, this rate was similar to the rate of resident success previously described.11,12 The high success rate (100\%) of both PEM and PICU fellows is likely due to the anesthesia rotations that are mandatory and usually completed early in fellowship. The success rate of PEM attending staff (76\%) was significantly lower than the published success of emergency medicine attending staff (98\%). ${ }^{9}$ This difference may be due in part to our small sample size and the fact that PEM attending staff are likely to face the most challenging intubations, leaving the easiest ones for their trainees. Conversely, this may also reflect the infrequency with which endotracheal intubations are performed by PEM attending staff when compared to their general emergency medicine colleagues.

Although the overall intubation success rate was high, this is only a crude marker of airway management 
skills. Other studies have found that although residents may be able to successfully intubate the trachea, ${ }^{13}$ they may have significant deficits in their overall airway management skills, including failure to set up monitors and suction, ${ }^{14}$ failure to adequately preoxygenate, ${ }^{15}$ inappropriate sizing of the mask and endotracheal tube,,$^{13,15}$ and poor laryngoscopy technique, ${ }^{14,15}$ skills that could not be assessed in this retrospective review.

Our study found that other resuscitation procedures were even more infrequent than intubation. During the 2-year study period, central line placement occurred only 7 times in the PED, which is considerably less than the yearly average of 22 times reported by a larger PED. ${ }^{16}$ Similarly, IO needle insertion was performed in only seven patients, four of whom were moribund and were eventually pronounced dead in the ED. There is scant literature addressing the frequency of IO insertion in tertiary level PEDs; our seemingly low number may be due to our highly skilled nurses, who regularly cannulate the veins of sick children, thus limiting the need for IO access. Nonetheless, interventions such as venous access should not be allowed to be the rate-limiting step in a resuscitation, and with the advent of powered IO insertion, which has been shown to be safe and effective, ${ }^{17}$ this is an acceptable alternative when vascular access cannot be readily obtained. Chest tube insertion and inotrope administration were also rare events. Thus, trainees in our department would not have enough exposure in the PED to learn central line and chest tube insertion, nor would attending physicians be able to adequately maintain their skills.

Although it is clear that clinical experience is deficient, what is needed to achieve competence is not fully understood. Anesthetists suggest that over 45 intubations are needed for novice intubators to achieve an $80 \%$ first attempt success rate. ${ }^{18,19}$ However, no data are available on what is needed to maintain skills for attending physicians. Cognitive knowledge alone is insufficient to ensure successful procedural skills. For example, although residents may perform well on cognitive knowledge testing such as the Pediatric Advanced Life Support (PALS) examination, their ability to perform all of the steps in resuscitation may be lacking. ${ }^{14}$

Our findings and those of others have important implications for training and skill maintenance. Clearly, more opportunities for practicing essential airway skills are required for both trainees and attending staff. Additional training opportunities may include extra time spent in anesthesia, simulation training, and advanced life support courses such as PALS and Advanced Trauma Life Support (ATLS). As well, it is important that the staff in the PED use difficult airway protocols and create a close working relationship with those in their hospital who have expertise in airway management such as anesthesia, otolaryngology, and pediatric intensive care. In addition to ongoing training, medical protocols, such as a sepsis bundle, can be used to further optimize care. PALS algorithms and resuscitation medications and dosages can be displayed prominently for quick reference when performing resuscitations.

\section{LIMITATIONS}

Missing or inaccurate medical records were a major limitation, as illustrated by the fact that the name and level of training were not documented in $19 \%$ of the intubations in the PED. Our method of case selection, patients admitted to the PICU and deaths in the ED, might have missed procedures such as tube thoracostomy for patients who were stable enough to be transferred to the surgical floor. This, however, would be a rare event as our hospital no longer has a standalone, step-down unit. The vast majority of children requiring stabilization in the $\mathrm{ED}$ will spend at least 24 hours in the PICU before being transferred to the floor. In addition, we used placement of the endotracheal tube in the trachea as our measure of successful intubation, which is only a crude marker of airway skill. As discussed, residents may be able to successfully intubate the trachea but still perform poorly in their overall airway management, ${ }^{15}$ which could not be assessed in a retrospective review. Finally, this was a single-centre study in a medium-sized tertiary level children's hospital that has 37,500 annual ED visits. Thus, our results may not be generalizable to other centres, in particular those of different sizes and varying levels of acuity.

\section{CONCLUSION}

Critical illness requiring aggressive medical resuscitation is a rare event in the PED. There are limited opportunities for trainees who need to acquire competency in pediatric resuscitation and PEM attending staff to maintain their skills. Further research 
is required to determine the number and type of resuscitation experiences required to achieve and maintain competency.

Acknowledgement: The authors wish to acknowledge Elaine Simpson, Instruction \& Reference Assistant, John W. Scott Health Sciences Library, University of Alberta, for helping out with our literature search related to our manuscript.

Competing interests: None declared.

\section{REFERENCES}

1. Donoghue AJ, Nadkarni VM, Elliott M, et al. Effect of hospital characteristics on outcomes from pediatric cardiopulmonary resuscitation: a report from the National Registry of Cardiopulmonary Resuscitation. Pediatrics 2006;118:9951001.

2. Chen EH, Cho CS, Shofer FS, et al. Resident exposure to critical patients in a pediatric emergency department. Pediatr Emerg Care 2007;23:774-8.

3. Gemke RJ, Weeteling B, van Elburg RM. Resuscitation competencies in paediatric specialist registrars. Postgrad Med 7 2007;83:265-7.

4. Schoenfeld PS, Baker MD. Management of cardiopulmonary and trauma resuscitation in the pediatric emergency department. Pediatrics 1993;91:726-9.

5. Chen EH, Shofer FS, Baren JM. Emergency medicine resident rotation in pediatric emergency medicine: what kind of experience are we providing? Acad Emerg Med 2004;11: 771-3.

6. Reis AG, Nadkarni V, Perondi MB, et al. A prospective investigation into the epidemiology of in-hospital pediatric cardiopulmonary resuscitation using the international Utstein reporting style. Pediatrics 2002;109:200-9.

7. Losek JD, Olson LR, Dobson JV, et al. Tracheal intubation practice and maintaining skill competency: survey of pediatric emergency department medical directors. Pediatr Emerg Care 2008;24:294-9.
8. Sakles JC, Laurin EG, Rantapaa AA, et al. Airway management in the emergency department: a one-year study of 610 tracheal intubations. Ann Emerg Med 1998;31:325-32.

9. Sagarin MJ, Barton ED, Chng YM, et al. Airway management by US and Canadian emergency medicine residents: a multicenter analysis of more than 6,000 endotracheal intubation attempts. Ann Emerg Med 2005;46:328-36.

10. Tayal VS, Riggs RW, Marx JA, et al. Rapid-sequence intubation at an emergency medicine residency: success rate and adverse events during a two-year period. Acad Emerg Med 1999;6:31-7.

11. Falck AJ, Escobedo MB, Baillargeon JG, et al. Proficiency of pediatric residents in performing neonatal endotracheal intubation. Pediatrics 2003;112(6 Pt 1):1242-7.

12. Sagarin MJ, Chiang V, Sakles JC, et al. Rapid sequence intubation for pediatric emergency airway management. Pediatr Emerg Care 2002;18:417-23.

13. White JR, Shugerman R, Brownlee C, et al. Performance of advanced resuscitation skills by pediatric housestaff. Arch Pediatr Adolesc Med 1998;152:1232-5.

14. Nadel FM, Lavelle JM, Fein JA, et al. Assessing pediatric senior residents' training in resuscitation: fund of knowledge, technical skills, and perception of confidence. Pediatr Emerg Care 2000;16:73-6.

15. Overly FL, Sudikoff SN, Shapiro MJ. High-fidelity medical simulation as an assessment tool for pediatric residents' airway management skills. Pediatr Emerg Care 2007;23: 11-5.

16. Chiang VW, Baskin MN. Uses and complications of central venous catheters inserted in a pediatric emergency department. Pediatr Emerg Care 2000;16:230-2.

17. Horton MA, Beamer C. Powered intraosseous insertion provides safe and effective vascular access for pediatric emergency patients. Pediatr Emerg Care 2008;24:347-50.

18. de Oliveira Filho GR. The construction of learning curves for basic skills in anesthetic procedures: an application for the cumulative sum method. Anesth Analg 2002;95:411-6.

19. Mulcaster JT, Mills J, Hung OR, et al. Laryngoscopic intubation: learning and performance. Anesthesiology 2003;98: $23-7$. 